\title{
Parametrization of CPW for Photonics Application with the Use of Closed Form Formulas
}

\author{
Sebastian Kula ${ }^{1 *}$ \\ 1 Institute of Mechanics and Applied Computer Science, Faculty of Mathematics, Physics and Technical Sciences, Kazimierz Wielki \\ University, 85-064 Bydgoszcz, Jana Karola Chodkiewicza 30, Poland \\ * Corresponding author, e-mail: skula@ukw.edu.pl
}

Received: 26 August 2019, Accepted: 19 January 2020, Published online: 26 March 2020

\begin{abstract}
In this paper parametrized analysis of CPW (Coplanar Waveguide) applied in photonics is presented. The analysis is based on analytical formulas, which were delivered and modified from conformal mapping technique, and also after review of available literature from analytical models for the conventional CPW. Adjustment of the already reported CPW formulas was performed to properly model thin films CPW structures in PIC (Photonic Integrated Circuit). Thus, a closed expressions were proposed to simulate basic parameters, like: per unit length (p.u.I.) $R$ (resistance), $L$ (inductance), $C$ (capacitance), $G$ (conductance) and propagation quantity like characteristic impedance Zo versus frequency. The CPW line dedicated for the PIC application, is based on InP (Indium Phosphide) substrate and with air or BCB (Benzocyclobutene) surroundings of the metallization on the top. The modified formulas are curvefitting approximations, with new fitting coefficients. Essential modifications are for p.u.I. C and p.u.I. L. Usefulness and effectiveness of the modified method was verified through results comparison with simulations in full-wave Electronic Design Automation software, with the circuit simulator QUCS and with real measurements, conducted with the use of PNA (Performance Network Analyzer). The paper novelty are new coefficients for analytical formulas, implementation of modified formulas in the octave code and model parametrization for geometrical parameters.
\end{abstract}

Keywords

coplanar waveguide, microwave, photonic integrated circuit, propagation quantities

\section{Introduction}

The Photonic Integrated Circuits are a constantly growing research and engineering area, these systems have great potential to eliminate in the future standard Integrated Circuit. The light propagating in the PIC enables faster data transfer, greater bandwidth, and at the same time less interference and loss because photons interfere with each other to a much lesser extent than electrons. At the same time, the disadvantage of the PIC systems is their size, which is micrometers, to nanometers, as is the case in semiconductor chips. The PIC miniaturization is currently a challenge in terms of both design and manufacturing technology. Another current challenge is the insufficient number of methods and tools for designing complex PIC. One of the method to overcome this challenge is derived from semiconductor electronics generic integration technology, it is technology, that allows for decoupling of design and technology by offering chip designers a small set of well-defined standardized building blocks with which they can design a broad range of application specific circuits [1]. The generic integration technology is becoming a standard and further development of the automation of designing Photonic Integrated Circuits and systems, tools and flows activities are ongoing at several software vendors, design houses and academia [2].

The increase of photonics applications in the area of engineering causes, that interest of designers and producers is moving from electronic Integrated Circuits, which are based on $\mathrm{Si}$, and is focusing on Photonic Integrated Circuits, which are based on such materials like InP, BCB or GaAs. The essential development is being observed in data transmission, this increasing interest of high speed transmission requires high quality RF-interconnections on PIC [3]. One of interconnect structure, which is very intensively used in electronic Integrated Circuits, is the CPW transmission line, hence such a big interest of this structure in the PIC devices. The CPW line is 
a relatively simple structure, it is cheap in manufacturing and due to this the line is very often used in circuits working in HF (High Frequency). At the same time CPW structures are more complex to be designed and simulated than for example MS (microstrip line), hence it is very crucial to prepare a robust, accurate, cost-efficient modeling method of the CPW in PIC devices. In order to create the model, which is robust in a wide range of the parameters, it is necessary to include completely the nonlinear phenomena [4].

In the paper the concept of knowledge transfer between silicon electronics and photonics is applied. Formulas typically used to model CPW in electronic IC (Integrated Circuit), like presented in [5-8] were modified and fitted to PIC structures. The modification of formulas has mainly curve-fitting character. New fitting coefficients are created and the modification is adjusted to typical PIC materials like InP and BCB. Obtained formulas are useful to properly simulate basic parameters, like per unit length (p.u.l.) $R$ (resistance), $L$ (inductance), $C$ (capacitance), $G$ (conductance) and propagation quantity like characteristic impedance $Z o$ versus frequency. The knowledge about these transmission line parameters and the propagation quantity is essential for PIC designers. New, modified formulas are closed expressions and can be relatively easy implemented in CAD (Computer Aided Design) software to model, simulate and design CPW in PIC structures.

Utility and functionality of the modified method were testified through results comparison with three commercial, full-wave EDA (Electronic Design Automation) software, ADS Momentum, Sonnet, AWRDE Microwave Office, with the free-software circuit simulator QUCS, and with real measurements, which were performed with the use of Performance Network Analyser. Verification was made in the microwave frequency range, up-to $40 \mathrm{GHz}$ for the simulation and also up-to $40 \mathrm{GHz}$ for the measurements data for very thin and narrow PIC CPW structures, in following dimension range: $0.0287 \leq 2 s / h_{3} \leq 0.24$; $0.0038 \leq t / h_{3} \leq 0.004 ; 0.0402 \leq g / h_{3} \leq 0.2$, where $t$ is the thickness of metallization, $2 s$ is the central metallization width and $h_{3}$ is the substrate layer height.

\section{Modified formulas}

The approach, presented in this work, is based on approximations, which are improvements of analytical formulas, already known in literature [5-8]. These improvements are the paper novelty. In this chapter the approach to obtain propagation quantities is depicted. The method is based on Transmission Line Theory and per unit length resistance $R$, inductance $L$, capacitance $C$, conductance $G$.
Per unit length $R, L, C, G$ values are calculated from modified analytical formulas and these values are used for calculation of the propagation quantity.

The structure of photonic CPW of interest is shown in Fig. 1. The CPW-type element has on the top metallization layer, performed from $\mathrm{Au}$ (with central width $2 s$, ground width $w_{g}$, gap $g$ and thickness $t$ ), air or BCB are surrounding metallization on top, below there are substrate layers, in our cases substrates are made from InP.

In order to create new, MF (modified formulas) correction coefficients are introduced, which values have been adjusted to fit EDA simulation data. The same EDA fitting procedure was used for all p.u.l. values. Formulas for line $C$ and $L$ are derived from publications by Gevorgian et al. [8], Heinrich [9] and Conformal-Mapping procedure [5]. These formulas are not only approximations but partially have also physical background and physical meaning. However new, modifying coefficients have approximation character only, they were matched with the results of EDA simulations.

The line capacitance of the CPW is $[8,9]$ :

$$
\begin{aligned}
& C=1.19 \cdot 4 \varepsilon_{0} \cdot \varepsilon_{e f 1} \frac{K\left(k_{5 C}\right)}{K\left(k_{5 C}^{\prime}\right)} \\
& k_{5 C}=\frac{s+\Delta}{s+g-\Delta} \cdot \sqrt{\frac{1-\left(\frac{s+g-\Delta}{s+g+w_{g}+\Delta}\right)^{2}}{1-\left(\frac{w+\Delta}{s+g+w_{g}+\Delta}\right)^{2}}}
\end{aligned}
$$

$k_{5 C}^{\prime}=\sqrt{1-k_{5 C}^{2}}$

where $K\left(k_{5 C}\right)$ and $K\left(k_{5 C}^{\prime}\right)$ are complete elliptic integrals of the first kind. In order to calculate complete elliptic integrals the built-in octave function is applied.

Coefficients $k_{5 C}$ are related to the geometrical dimensions. Coefficient 1.19 in Eq. (1) is the paper novelty. In this approximation the effective dielectric permittivity is given by [10]:

$\varepsilon_{e f 1}=1+\left(\varepsilon_{1}-1\right) q_{1}+\left(\varepsilon_{3}-1\right) q_{3}$

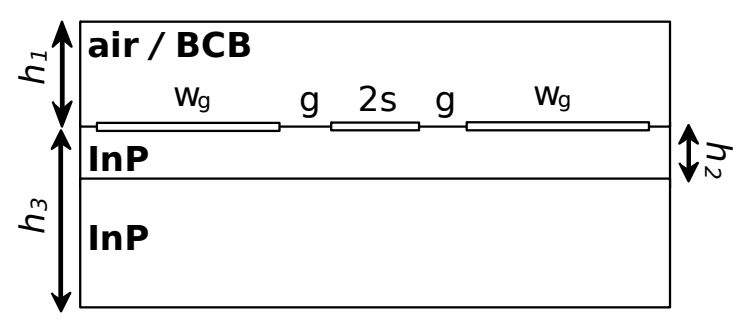

Fig. 1 The cross-section of the CPW, where $2 s$ is the central metallization width, $w_{g}$ ground width, $g$ gap and $h_{1}, h_{2}, h_{3}$ are layers heights. 
where $\varepsilon_{1}$ is in paper's analysis permittivity of the air or BCB and $\varepsilon_{3}$ of InP. Factors $q_{1}$ and $q_{3}$ are calculated from $[10,11]$ :

$q_{i}=\frac{1}{2} \frac{K\left(k_{i}\right)}{K\left(k_{i}^{\prime}\right)} \frac{K\left(k_{5 C}^{\prime}\right)}{K\left(k_{5 C}\right)}, \quad i=1,3$

$$
k_{i}=\frac{\sinh \left(\frac{\pi(s+\Delta)}{2 h_{i}}\right)}{\sinh \left(\frac{\pi(s+g-\Delta)}{2 h_{i}}\right)} .
$$

$$
\sqrt{\frac{1-\frac{\sinh ^{2}\left(\frac{\pi(s+g-\Delta)}{2 h_{i}}\right)}{\sinh ^{2}\left(\frac{\pi\left(s+g+w_{g}+\Delta\right)}{2 h_{i}}\right)}}{1-\frac{\sinh ^{2}\left(\frac{\pi(s+\Delta)}{2 h_{i}}\right)}{\sinh ^{2}\left(\frac{\pi\left(s+g+w_{g}+\Delta\right)}{2 h_{i}}\right)}}}
$$

$i=1,3$

The parameter $\Delta$ takes into account thickness of the metallization $t[8]$ :

$\Delta=\frac{t}{2 \pi \varepsilon_{e}}\left[1+\ln \left(\frac{8 \pi s}{t}\right)\right]$

where $\varepsilon_{e}$ is the largest permittivity of the layers in an immediate contact with the metallization, $\varepsilon_{e}=\max \left\{\varepsilon_{1}, \varepsilon_{3}\right\}$.

The line inductance of CPW is [8]:

$L=0.88 \cdot \frac{\mu_{0}}{4} \frac{K\left(k_{5 L}^{\prime}\right)}{K\left(k_{5 L}\right)}$

where $K\left(k_{5 L}\right)$ and $K\left(k_{5 L}^{\prime}\right)$ are complete elliptic integrals with coefficients $k_{5 L}$ related to the geometrical dimensions and skin depth. Coefficient 0.88 is the paper novelty. Coefficients of elliptic integrals are obtained from $[8,11]$ :

$k_{5 L}=\frac{s-2.1 \delta_{s}}{s+g+2.1 \delta_{s}} \cdot \sqrt{\frac{1-\left(\frac{s+g+2.9 \delta_{s}}{s+g+w_{g}-\delta_{s}}\right)^{2}}{1-\left(\frac{s-2.9 \delta_{s}}{s+g+w_{g}-2.9 \delta_{s}}\right)^{2}}}$

$k_{5 L}^{\prime}=\sqrt{1-k_{5 L}^{2}}$

where $s-\delta_{s}$ is effective width and $\delta_{s}$ is skin depth, $\delta_{s}=1 / \sqrt{\left(\pi \mu_{0} \sigma_{m} f\right)}, \mu_{0}$ is the magnetic constant of vacuum, $\sigma_{m} \mathrm{~S} / \mathrm{m}$ is conductivity of the metallization. Coefficients 2.1 and 2.9 are modifying novelty in this formula.

The p.u.l. $G$ conductance is calculated in typical way and is based on already calculated line capacitance Eq. (1):

$$
G=C \cdot 2 \pi \cdot f \cdot \tan \delta
$$

where $\tan \delta$ is the loss tangent of the InP layer.
The p.u.l. resistance $R$ is given by:

$$
R=\frac{1}{2 s \sigma_{m} \delta_{s}\left\{1-\exp \left(-t / \delta_{s}\right)\right\}}
$$

where $\delta_{s}$ is the skin depth, $\delta_{s}=1 / \sqrt{\left(\pi \mu_{0} \sigma_{m} f\right)}, \mu_{0}$ is the magnetic constant of vacuum, $\sigma_{m} \mathrm{~S} / \mathrm{m}$ is the conductivity of the metallization.

At this point of the approach presentation it can be considered, that p.u.l. $C, L, G$ and $R$ are known and calculated, hence the propagation quantity like characteristic impedance $Z o$ is calculated as follows:

$Z o=\sqrt{\frac{R+j \omega L}{G+j \omega C}}$.

\section{Formulas validation}

The proposed, modified formulas were compared to EDA data and to measurements. The comparison was made with EDA (ADS Momentum, Sonnet, AWRDE Microwave Office) results in the specified limits of the dimensions, up-to $40 \mathrm{GHz}$. Estimated maximum relative errors for $0.0287 \leq 2 s / h_{3} \leq 0.2400,0.0038 \leq t / h_{3} \leq 0.0040$, $0.0402 \leq g / h_{3} \leq 0.2000$ are given below:

- at about $40 \mathrm{GHz}$, modified formulas to EDA

$$
\operatorname{Max}\left(\frac{\Delta Z o}{Z o}\right)=4.6 \%
$$

- at about $10 \mathrm{GHz}$, modified formulas to EDA

$$
\operatorname{Max}\left(\frac{\Delta Z o}{Z o}\right)=2.8 \% \text {. }
$$

The measurements were made up-to $40 \mathrm{GHz}$, with the use of two-port probe station, Performance Network Analyzer PNA Agilent Technologies N5230A and TRL (Throughreflect-line) calibration. The direct measurements were in the form of scattering parameters ( $S$-parameters) $S_{11}, S_{12}$, $S_{21}, S_{22}$. In order to obtain the characteristic impedance $Z o$ from the measured results the QUCS circuit simulator was applied. As presented in Fig. 2 with the use of the QUCS SPfile $X_{1}$ component, which enables to load $s 2 p$ files (touchstone format) containing $S$-parameters, with two

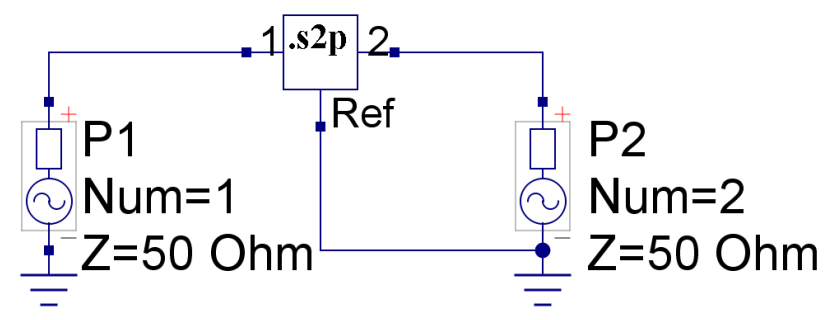

Fig. 2 The schematic in the QUCS software. Extraction of the characteristic impedance $Z o$ of the CPW from the measured results, which were obtained with the use of the PNA device. 
power sources $P_{1}$ and $P_{2}$, which are required for the twoport $S$-parameter simulation and with the QUCS twoport() function, which converts a two-port matrix from one representation into another one, the $S$-parameters were transformed into $A B C D$ matrix. The characteristic impedance was extracted with the use of $B$ and $C$ parameters of $A B C D$ matrix, which describes the two-port network. The formula for the characteristic impedance is as follows:

$Z o=\sqrt{\frac{B}{C}}$.

The comparison between measurements, EDA and MF was made at high frequency from $25 \mathrm{GHz}$ up-to $40 \mathrm{GHz}$ and this comparison is presented at Fig. 3. The comparison was made on the example 1: $2 s=30.1 \mathrm{um}$, $w_{g}=52.17 \mathrm{um}, g=25.23 \mathrm{um}, t=1 \mathrm{um}, h_{3}=250 \mathrm{um}$, $\varepsilon_{1}=1$ (air), $\varepsilon_{2}=\varepsilon_{3}=12.4$ (InP), $\sigma_{m}=41 \mathrm{MS} / \mathrm{m}$ (Au). For the same example 1 the results comparison from $1 \mathrm{GHz}$ up to $40 \mathrm{GHz}$ is presented in Fig. 4.

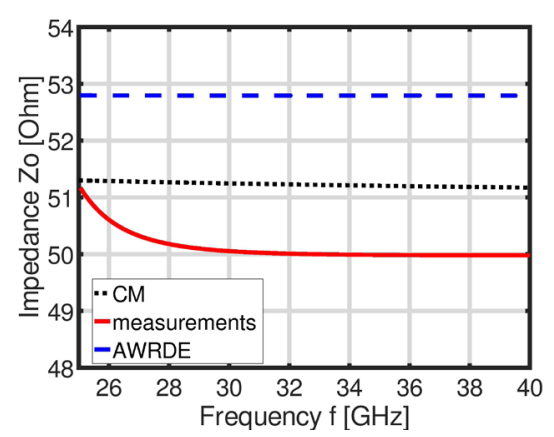

Fig. 3 The comparison between measurements, EDA (AWRDE) and modified formulas (MF) for the characteristic impedance $Z o$ versus frequency in high frequency range from $25 \mathrm{GHz}$ up-to $40 \mathrm{GHz}$, results for example 1 .

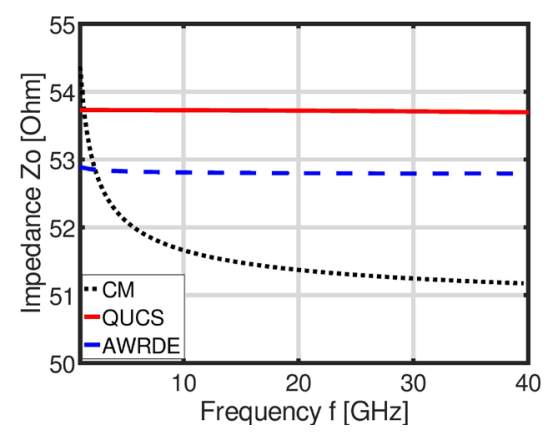

Fig. 4 The comparison between EDA (AWRDE), the circuit simulator QUCS and modified formulas (MF) for the characteristic impedance Zo versus frequency in frequency range from $1 \mathrm{GHz}$ up to $40 \mathrm{GHz}$, results for example 1 .

\section{Parametrization of CPW}

Parametrization is an essential for sensitivity analysis and optimization. During the technological process of the CPW fabrication, it is a standard that some inaccuracy appears, hence there is a necessity of the sensitivity analysis, with the use of parametrized models. The optimization is crucial during the $\mathrm{CPW}$ design routine and also requires parametrized models.

The code in octave enables to conduct parametrization in relatively easy way. The only additional requirement, in comparison to the model with the constant settings of the parameters, is to set an input vector with parametrized values of the parameter. The code sweeps the parameter with accordance to the vector values and without additional modifications the code calculates the characteristic impedance versus the parameter value and versus the frequency. Fig. 5 presents the top view of the CPW and geometrical parameters, which can be parametrized.

In the paper results of the $w_{g}$ (ground width) parametrization is presented on Fig. 6. As it can be observed the analysis was done in the range of the $w_{g}$ from $30 \mathrm{um}$ up-to $180 \mathrm{um}$, at two frequencies $10 \mathrm{GHz}$ and $30 \mathrm{GHz}$. The widther ground strip $w_{g}$ results in the lower characteristic impedance, which is related to the higher p.u.l. capacitance for the widther $w_{g}$ strip, the higher frequency also results in the lower characteristic impedance.

\section{Conclusions}

In the paper the analysis of the parametrized model, which represents the CPW line based on InP substrates and $\mathrm{Au}$ metallization is depicted. The model fully verifies the CPW in the frequency range from $1 \mathrm{GHz}$ to $40 \mathrm{GHz}$. The analysis is focused on the characteristic impedance and its dependency on frequency and ground metallization width.

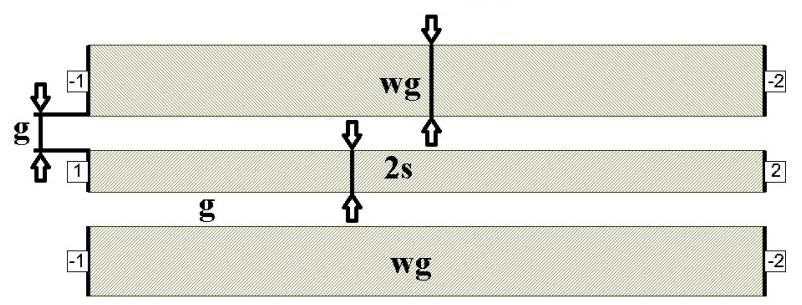

Fig. 5 The top view of the CPW, where geometrical parameters like: $2 s$ the central metallization width, $w_{g}$ ground width, $g$ gap are indicated. For all these parameters it is feasible to conduct parametrization analysis with the use of the code in octave. 


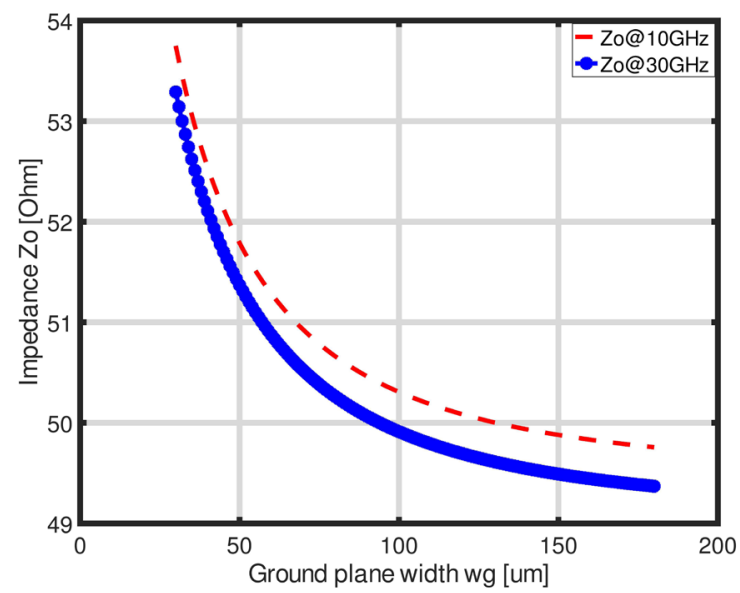

Fig. 6 The parametrization results of $w_{g}$ (ground width) versus characteristic impedance $Z o$. The analysis conducted at $10 \mathrm{GHz}$ and at $30 \mathrm{GHz}$.

The proposed model was verified with EDA simulations and measurements. There is a good agreement between MF and EDA data and between MF and measurements data.

\section{References}

[1] Smit, M., Williams, K., van der Tol, J. "Past, present, and future of InP-based photonic integration", APL Photonics, 4(5), Article number: 050901, 2019.

https://doi.org/10.1063/1.5087862

[2] The JePPIX consortium "JePPIX roadmap 2018, the road to a multi-billion Euro market in integrated photonics", [online] Available at: http://wipe.jeppix.eu/public/images/JePPIXRoadmap-may2018_FINAL.pdf [Accessed: 15 August 2019]

[3] Besten, J. H., Pahuta, R., Roodnat, A. W., Leijtens, X. J. M., Smit, M. K. "Rf-interconnections on InP", In: 5th annual symposium of the IEEE/LEOS Benelux Chapter, Delft, Netherlands, 2000, pp. 75-78. [online] Available at: http://repository.tue.nl/590047 [Accessed: 20 August 2019]

[4] Kula, S., Lup, A. "Parametrized reduced model of RF MEMS capacitive switch", In: 2017 10th International Symposium on Advanced Topics in Electrical Engineering (ATEE), Bucharest, Romania, 2017, pp. 529-532.

https://doi.org/10.1109/ATEE.2017.7905180

[5] Wheeler, H. A. "Transmission-Line Properties of Parallel Wide Strips by a Conformal-Mapping Approximation", IEEE Transaction on Microwave Theory and Techniques, 12(3), pp. 280-289, 1964. https://doi.org/10.1109/TMTT.1964.1125810

[6] Liao, C. L., Tu, Y. M., Ke, J. Y., Chen, C. H. "Transient propagation in lossy coplanar waveguides", IEEE Transaction on Microwave Theory and Techniques, 44(12), pp. 2605-2611, 1996.

https://doi.org/10.1109/22.554610
The created formulas can be used for fast, preliminary, based on low-computing hardware resources, design of the CPW lines for photonic systems. The resulting methods were implemented in the octave programming language, hence they are relatively easy to adapt in the matlab, and thus the code is potentially available for application in many programming languages and CAD design tools. The main concept of the work was to propose formulas and apply them in the generic integration technology, by creating, on their basis, standardized blocks representing the photonic CPW line. The formulas are in the form, which enables to apply them in the generic integration technology blocks. The generic integration technology is increasingly applied by PIC designers and is available in many EDA tools.

\section{Acknowledgement}

The part of the research presented in this paper has been done in the frame of the FP7-ICT PARADIGM (Photonic Advanced Research and Development for Integrated Generic Manufacturing).

[7] Benevent, E., Viala, B., Michel, J. P. "Analytical Modeling of Multilayered Coplanar Waveguides Including Ferromagnetic Thin Films on Semiconductor Substrates", IEEE Transaction on Microwave Theory and Techniques, 58(3), pp. 645-650, 2010. https://doi.org/10.1109/TMTT.2010.2040336

[8] Gevorgian, S., Martinsson, T., Deleniv, A., Kollberg, E., Vendik, I. "Simple and accurate dispersion expression for the effective dielectric constant of coplanar waveguides", IEE Proceedings Microwaves, Antennas and Propagation, 144(2), pp. 145-148, 1997. https://doi.org/10.1049/ip-map:19970843

[9] Heinrich, W. "Quasi-TEM description of MMIC coplanar lines including conductor-loss effects", IEEE Transaction on Microwave Theory and Techniques, 41(1), pp. 45-52, 1993. https://doi.org/10.1109/22.210228

[10] Carlsson, E., Gevorgian, S. "Conformal mapping of the field and charge distributions in multilayered substrate CPWs", IEEE Transaction on Microwave Theory and Techniques, 47(8), pp. 1544-1552, 1999. https://doi.org/10.1109/22.780407

[11] Ghione, G., Naldi, C. U. "Coplanar Waveguides for MMIC Applications: Effect of Upper Shielding, Conductor Backing, FiniteExtent Ground Planes, and Line-to-Line Coupling", IEEE Transaction on Microwave Theory and Techniques, 35(3), pp. 260-267, 1987. https://doi.org/10.1109/TMTT.1987.1133637 